\title{
Diffuse Axonal Injury
}

\author{
Maj V Maurya", Brig AKS Bairaria ${ }^{+}$Brig CM Adya, vsm", Capt YS Rajesh ${ }^{* *}$
}

MJAFI 2006; 62 : 277-279

Key Words : Axonal injury

\section{Introduction}

$\mathrm{D}$ iffuse axonal injury (DAI) is a result of traumatic deceleration injury and a frequent cause of persistent vegetative state in patients. DAI is the most significant cause of morbidity in patients with traumatic brain injuries, which commonly result from highspeed motor vehicle accidents. We report one such case.

\section{Case Report}

A 27 year-old serving soldier was brought in a comatose condition to a service hospital after a road traffic accident. On examination, respiratory rate was 34 per minute, pulse was 84 beats per minute and blood pressure was 130/84 $\mathrm{mmHg}$. He had multiple abrasions over left side of face. No other external injury was noted. His Glasgow Coma score was $5 / 15$, right pupil was constricted and left was dilated with no reaction to light. Plantars were bilaterally extensor and deep tendon jerks absent.

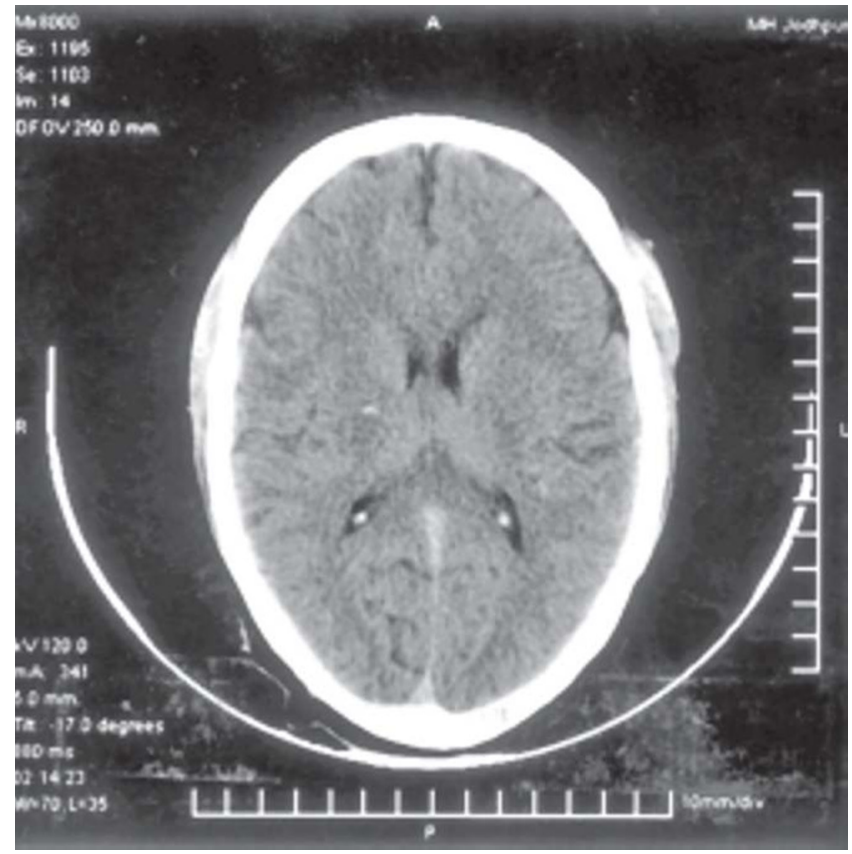

Fig. 1 : NCCT head shows a tiny hemorrhagic focus in the right basal ganglia
Non contrast computed tomography (NCCT) head showed tiny haemorrhagic foci in the right basal ganglia (Fig 1) and brain stem with no evidence of any extradural or subdural haematoma. There was no bony injury. Clinical profile and computed tomography (CT) findings were suggestive of diffuse axonal injury. T1W, T2W and fluid-attenuated inversion recovery (FLAIR) MRI was done which showed altered signal intensity in the basal ganglia bilaterally (Fig 2 and 3), posterior part of body of corpus callosum (Fig 4) and cerebral peduncle of midbrain appearing hyperintense in $\mathrm{T} 2 \mathrm{~W}$ images and FLAIR sequences and hypointense in T1W images. These findings were consistent with diffuse axonal injury. Mild bilateral frontal subdural collection was also noted (Fig 2).

Patient developed cheyne stokes respiration on second day and was put on ventilatory support. Patient was removed from the ventilator on seventh day. There was no change in his neurological status with Glasgow Coma Scale (GCS)

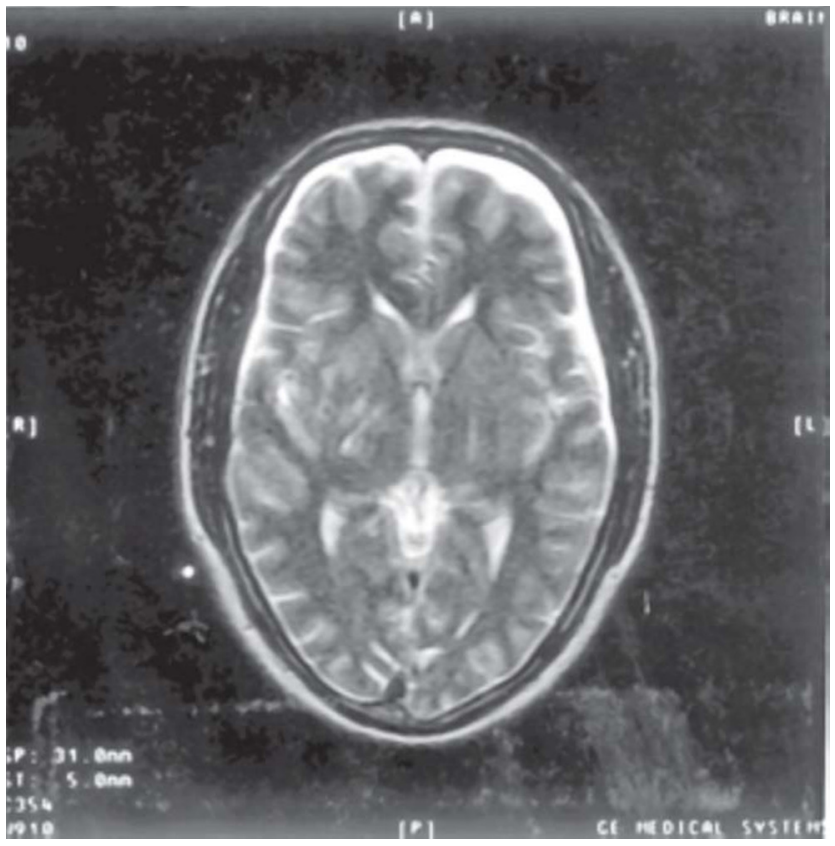

Fig. 2: T2W axial MRI showing patchy hyperintensities in the basal ganglia bilaterally and mild bilateral frontal subdural collection

\footnotetext{
* Graded Specialist (Radiology), 151 BH, C/o 99 APO, +DDMS, HQ4 Corps (Med) C/o 99 APO, "Comdt, MH Ambala Cantt, Haryana, ${ }^{* *}$ RMO, 17 RR C/o 99 APO.
}

Received : 12.06.2004; Accepted : 05.01.2006 


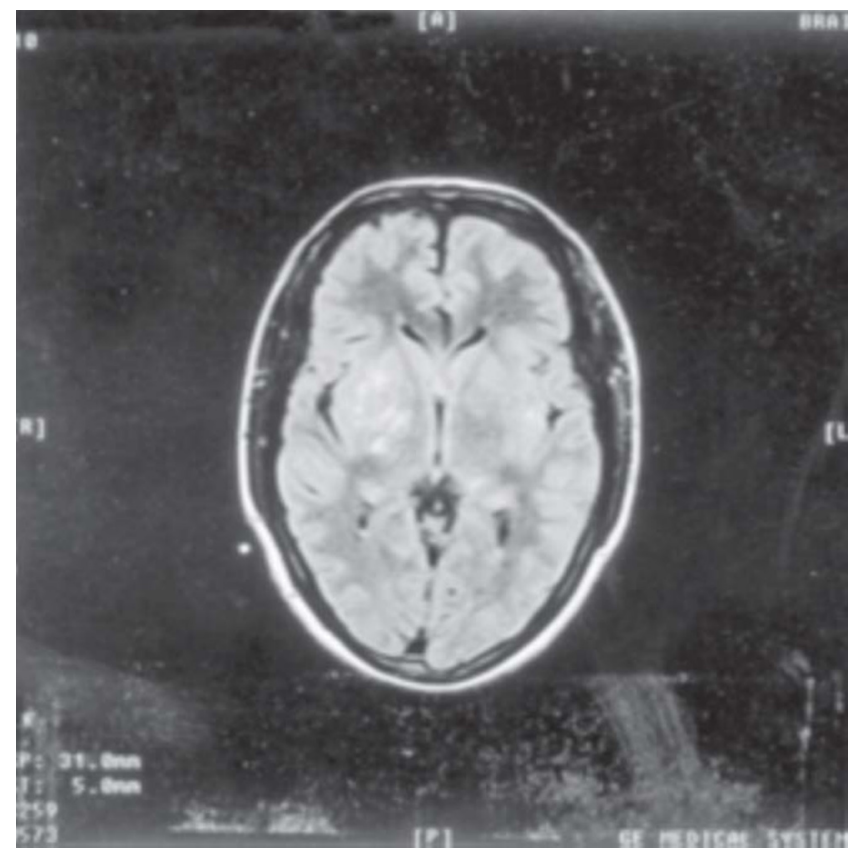

Fig. 3 : FLAIR sequence demonstrating multiple foci of abnormal increased signal in the basal ganglia bilaterally

remaining 5/15. Right pupil was constricted and left pupil dilated and fixed. Deep tendon jerks became brisk with plantars bilaterally extensor. Patient was air lifted to a tertiary care hospital in persistent vegetative state for further management.

\section{Discussion}

Diffuse axonal injury also called shear injury is one of the most frequent types of primary injury that is seen in patients with severe head trauma. Pathologically it leads to a brain injury, with features of axon swelling, axon breaking and ball retracting.

DAI represents approximately half of all intra-axial traumatic lesions. It has no sex predilection and can occur at any age. Some studies suggest that DAI may occur in utero if a pregnant woman is subjected to sufficient force [1].

DAI typically consists of multiple, bilateral, small, focal lesions throughout the white matter, mainly at the greywhite junction. The lesions are located in three characteristic areas: corpus callosum, brain stem (in particular the dorsolateral region and the cerebral peduncle), and lobar white matter (frontal and temporal). Corpus callosum lesions are located mainly in the posterior body and splenium. Three stages of involvement have been described by Adams et al [2] according to the anatomic location of the lesions with stage I involving the parasagittal regions of the frontal lobes, periventricular temporal lobes and occasionally, the parietal and occipital lobes, internal and external capsules and cerebellum. Stage II involving the corpus callosum, in addition to the white matter areas in stage I. The involvement of the corpus callosum carries a poor

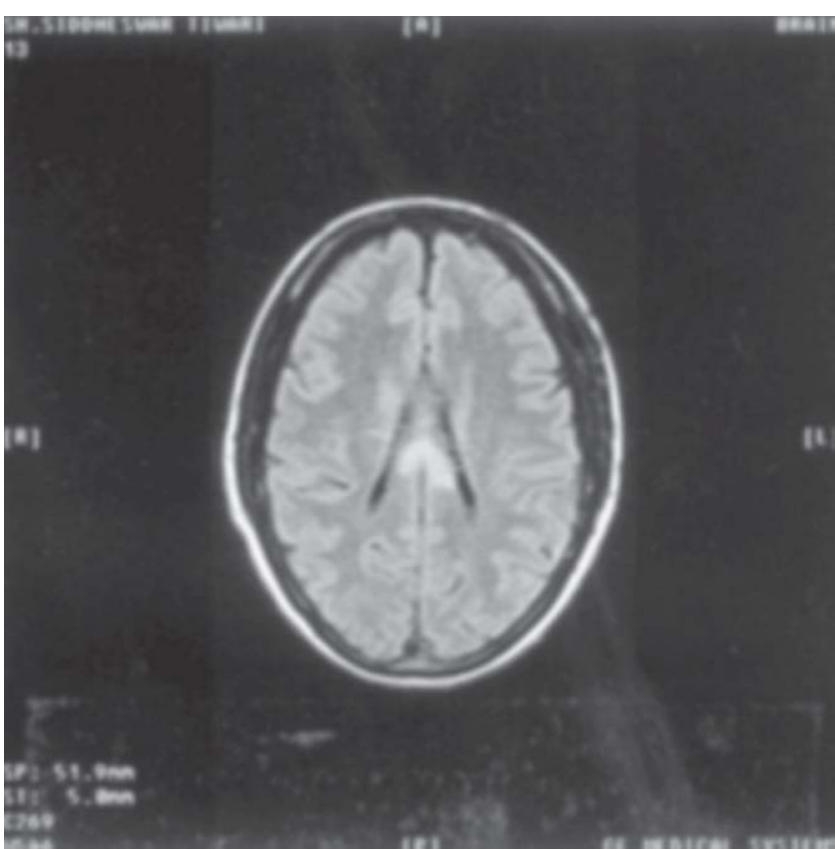

Fig. 4 : FLAIR sequence demonstrating oedema within the posterior part body of the corpus callosum secondary to traumatic shearing injury

prognosis. Stage III involves the areas associated with stage II and brainstem. A predilection for the superior cerebellar peduncles, medical lemnisci and corticospinal tracts is found.

Delayed CT scan may be helpful in demonstrating oedema or atrophy, which are later findings. MRI is more sensitive for subtle soft-tissue abnormalities than $\mathrm{CT}$ and is the imaging modality of choice for demonstrating DAI, however CT is commonly available. According to Teasdale [3], CT remains the "mainstay of investigation for acute head injury". The most common MRI finding is multifocal areas of abnormal signal (bright on T2-weighted images and FLAIR sequence) at the white matter in the temporal or parietal corticomedullary junction, basal ganglia or in the splenium of the corpus callosum. Other areas that are frequently abnormal include the dorsolateral rostral midbrain and the corona radiata.

Clinically DAI results in instantaneous loss of consciousness, and most patients $(>90 \%)$ remain in a persistent vegetative state, since brainstem function remains unaffected. DAI rarely causes death. The chance that a patient will remain in a persistent vegetative state is greater when lesions are observed in the supratentorial white matter, corpus callosum, and corona radiata. The presence of haemorrhage in DAI-type lesions and the association with traumatic spaceoccupying lesions is a poor prognostic sign. Isolated non haemorrhagic DAI-type lesions have better prognosis [4]. The prognosis also worsens as the number of lesions increase. For almost $10 \%$ of patients who experience a 
return to any form of normal function, this improvement will be seen within the first year. DAI lesions can result in deficit in information transfer between the two sides of the corpus callosum, commonly resulting in auditory deficits.

In conclusion, DAI is a significant medical problem because of the high level of debilitation of the patient, the stress for the patient's family and the staggering medical cost of sustaining an individual in this state.

\section{Conflicts of Interest}

None identified

\section{References}

1. Yamamoto T, Koeda T, Ishii S, Takeshita K. A patient with cerebral palsy whose mother had a traffic accident during pregnancy: a diffuse axonal injury? Brain Dev 1999; 21: 334-6.

2. Adams JH, Jennett B, Mc Lellan DR, et al. The neuropathology of the vegetative state after head injury. J Clin Pathol 1999; 52: 804-6.

3. Teasdate GM. Head injury. J Neurol Neurosurg Psychiatry 1995; 58: 526-39.

4. Paterakis K, Karantanas AH, Komnos A, Volikas Z. Outcome of patients with diffuse axonal injury: the significance and prognostic value of MRI in the acute phase. J Trauma 2000; 49: 1071-5. 